\section{CPS-296 IMPACT OF THE COVID-19 PANDEMIC ON CLINICAL LUNG CANCER MANAGEMENT}

${ }^{1}$ EM Barreiro Fernandez*,${ }^{2} \mathrm{C}$ Martinez Diaz, ${ }^{2} \mathrm{FJ}$ Salmeron Navas, ${ }^{2} \mathrm{~S}$ Fenix Caballero ${ }^{2}$ EJ Alegre-Del Rey, ${ }^{2} \mathrm{M}$ Dominguez Cantero. ${ }^{1}$ Hospital Universitario Puerto Real, Servicio De Farmacia, Cadiz, Spain; ${ }^{2}$ Hospital Universitario Puerto Real, Farmacia Hospitalaria, Cadiz, Spain

\subsection{6/ejhpharm-2021-eahpconf.128}

Background and importance The SARS-CoV-2 pandemic could have changed the clinical management of cancer patients because of travel restrictions, overloading of hospital systems and disruption of treatment. Lung cancer patients constitute a vulnerable population due to the particular risk of their disease, chemotherapy or immunotherapy.

Aim and objectives To analyse disease management and the clinical impact of the COVID-19 pandemic on non-small cell lung cancer (NSCLC) patients receiving intravenous treatment during the social isolation period compared with the normal situation.

Material and methods This retrospective observational cohort study included a 2:1 random sample of NSCLC patients in the 'COVID cohort' (patients in isolation February 2020 to June 2020) and the 'no COVID cohort' (patients treated between February 2019 and June 2019). Collected variables from digital clinical history were age, sex, stage, previous lines, type of treatment, number of medical visits and telephone consults, cycles received, worsening of performance status (PS), respiratory infection (COVID-19 and others), delays, therapeutic rest break, disease progression and deaths.

Results COVID cohort (CC): 40 patients, 31 (78\%) men; mean 67 years (59-84). Cancer stage: IV (69\%), IIIB (28\%), IIIA $(2 \%)$ and IIIC (1\%). $12(30 \%)$ patients had not received lines previously. $38 \%$ of the population received immunotherapy. Median number of medical visits was 3 (14-1) and median number of telephone consults was 3 (1-8). Median number of cycles was 4 (1-16). PS 0 (58\%) and PS 1 (42\%). No patient had COVID-19.

No COVID cohort' (NCC): 20 patients, 15 (75\%) men, mean 67 years (54-85). Cancer stage: stage IV (75\%), IIIB (25\%). All patients had received lines previously and none had received immunotherapy. Median medical visits was 7 (311) with no telephone consults. Median number of cycles was 3 (1-11). PS $0(70 \%)$ and PS1-2 (30\%). The rest of the variables are shown in table 1 .

Conclusion and relevance In spite of the limitations of the study, the new strategies of clinical management during the COVID-19 pandemic (telephone consults and therapeutic tire) did not appear to affect disease progression and NSCLC patient survival although worsening of performance status was observed.

\begin{tabular}{lllll} 
Abstract 4CPS-296 Table 1 & & \\
\hline & CC (\%) & NCC (\%) & RAR 30\% (95\% CI) & p value \\
\hline Worsening PS & 30 & 0 & 15.8 to 44.2 & $<0.05$ \\
Therapeutic rest break & 35 & 0 & 20.2 to 49.7 & $<0.05$ \\
Delays & 100 & 30 & -90.1 to -49.9 & $<0.05$ \\
Respiratory infections & 10 & 15 & -13.2 to 23.2 & $>0.05$ \\
Disease progression & 30 & 45 & -11.2 to 41.2 & $>0.05$ \\
Deaths & 18 & 25 & -14.8 to 29.8 & $>0.05$ \\
\hline
\end{tabular}

\section{REFERENCES AND/OR ACKNOWLEDGEMENTS}

Conflict of interest No conflict of interest

\section{CPS-297 SPINAL CORD APLASIA CAUSED BY 6- MERCAPTOPURINE IN A CAUCASIAN GIRL WITH ACUTE LYMPHOBLASTIC LEUKAEMIA AND HOMOZYGOUS MUTATION IN NUDIX HYDROLASE 15: CASE REPORT}

${ }^{1}$ LE Pineda Lancheros* ${ }^{2} \mathrm{C}$ Pérez Ramírez, ${ }^{3} \mathrm{~N}$ Moreno Toro, ${ }^{4} \mathrm{~A}$ Espinosa Rodriguez, ${ }^{4}$ A Sánchez Martín. ${ }^{1}$ Pharmacogenetics Unit, Pharmacy Service, University Hospital Virgen De Las Nieves, Granada, Spain; ${ }^{2}$ Pharmacy Service, University Hospital Virgen Macarena, Granada, Spain; ${ }^{3}$ Paediatric Oncohaematology, University Hospital Virgen De Las Nieves, Granada, Spain; ${ }^{4}$ Pharmacy Service, University Hospital Virgen De Las Nieves., Granada, Spain

\subsection{6/ejhpharm-2021-eahpconf.129}

Background and importance 6-mercaptopurine (6-MP) is an anticancer and immunosuppressive agent used as part of the therapeutic strategy in acute lymphoblastic leukaemia (ALL), in the induction, consolidation and maintenance phases. However, it may cause life threatening myelotoxicity that is commonly associated with polymorphisms in genes involved in its metabolism (thiopurine methyltransferase (TPMT) and nudix hydrolase 15 (NUDT15)).

Aim and objectives To describe the clinical case of a Caucasian girl, aged 2 years, diagnosed with B-ALL (intermediate risk of hyperdiploidy in cytogenetics (DNI index 1.27) and MRD on day +15 of $1.4 \%$, CNS-1) that presented prolonged myelotoxicity under the LAL-SEHOP-PETHEMA-2013 treatment protocol.

Material and methods During the induction phase, prolonged spinal cord aplasia occurred with the complication of sepsis due to Staphylococcus epidermidis, which required intensive care unit support. Subsequently, she presented sepsis due to Pseudomonas, a granuloma in the reservoir area that requires channelling of a peripheral access central catheter type PICC. TPMT polymorphisms $(* 2, * 3 \mathrm{~A}, * 3 \mathrm{~B}$ and $* 3 \mathrm{C})$ were studied without alterations. Therefore, the consolidation phase continued, and she presented with aplasia, febrile neutropenia, respiratory infection and central venous catheter infection. The dose of $6-\mathrm{MP}$ was reduced to $10 \%$. In the reinduction phase she also showed aplasia, febrile neutropenia and mucocutaneous infection by Candida dubliniensis. During maintenance, methotrexate and 6-MP were suspended several times, and 6MP was resumed at $3 \%$ of the dose. She received multiple transfusions of red blood cells and platelets.

To understand the toxicity manifested by the patient and considering the update of the pharmacogenetic guide for thiopurines of the Clinical Pharmacogenetics Implementation Consortium, real time PCR genotyping by Taqman was performed for NUDT15-rs116855232 gene polymorphism.

Results Although initial genetic testing revealed that the patient carried the TPMT wild-type allele, the patient had prolonged spinal cord aplasia and multiple infectious complications. Subsequent analysis revealed that the patient carried the rs116855232-TT genotype (frequency in Europeans 0.000004). This polymorphism is associated with potentially fatal myelosuppression (evidence level 1A), which explains the toxicity manifested.

Conclusion and relevance This case shows the relevance of implementing pharmacogenetics studies (TPMT and NUDT15 